\title{
Modeling of Progesterone Release from Poly(3-Hydroxybutyrate) (PHB) Membranes
}

\author{
Analia I. Romero, ${ }^{1}$ José M. Bermudez ${ }^{1,2}$ Mercedes Villegas, ${ }^{1}$ María F. Dib Ashur, ${ }^{1}$ \\ Mónica L. Parentis, ${ }^{1}$ and Elio E. Gonzo ${ }^{1}$
}

Received 11 June 2015; accepted 2 September 2015; published online 29 September 2015

\begin{abstract}
Poly(3-hydroxybutyrate) (PHB) biodegradable polymeric membranes were evaluated as platform for progesterone (Prg)-controlled release. In the design of new drug delivery systems, it is important to understand the mass transport mechanism involved, as well as predict the process kinetics. Drug release experiments were conducted and the experimental results were evaluated using engineering approaches that were extrapolated to the pharmaceutical field by our research group. Membranes were loaded with different Prg concentrations and characterized by scanning electron microscopy (SEM), differential scanning calorimetry (DSC), and Fourier transform infrared spectroscopy (FTIR). SEM images showed that membranes have a dense structure before and after the progesterone addition. DSC and FTIR allowed determining the influence of the therapeutic agent in the membrane properties. The in vitro experiments were performed using two different techniques: (A) returning the sample to the receptor solution (constant volume of the delivery medium) and (B) extracting total volume of the receptor solution. In this work, we present a simple and accurate "lumped" second-order kinetic model. This lumped model considers the different mass transport steps involved in drug release systems. The model fits very well the experimental data using any of the two experimental procedures, in the range $0 \leq t \leq \infty$ or $0 \leq M_{t} \leq M_{\infty}$. The drug release analysis using our proposed approaches is relevant for establishing in vitro-in vivo correlations in future tests in animals.
\end{abstract}

KEY WORDS: biodegradable polymers; controlled release/delivery; drug transport; mathematical models; membranes.

\section{INTRODUCTION}

Controlled-release systems have been developed to allow continuous and sustained drug release in the body, to protect drug from physiological degradation or elimination, to improve patient compliance, and to enhance quality control in manufacturing of drug products. When designing controlledrelease systems, it is important to identify and understand particular mechanisms involved in release processes. Often, more than one mechanism is involved at a given time or different mechanisms may dominate at different stages of the delivery processes.

In the design of new drug delivery systems, it is important to understand the mass transport mechanism involved in the release, as well as quantitatively predict the kinetics of the process. Thereby, through suitable mathematical models, it is possible to estimate the effect of system design parameters on the drug release kinetics (1-5).

\footnotetext{
${ }^{1}$ Facultad de Ingeniería, Instituto de Investigaciones para la Industria Química (INIQUI-CONICET), Universidad Nacional de Salta, Av. Bolivia 5150, A4408FVY, Salta Capital, Argentina.

${ }^{2}$ To whom correspondence should be addressed. (e-mail: jbermudez@unsa.edu.ar)
}

The vast majority of theoretical models are based on diffusion equations. The phenomenon of diffusion is intimately connected to the structure of the material through which the diffusion takes place; thus, the morphology of the polymeric materials should be considered for a successful model. There are some interesting reviews addressing these aspects in controlled-release formulations (1,6-10).

The mechanisms of drug release could be classified into diffusion, chemically or swelling-controlled processes (11-13). Drug transport through the polymer bulk, known as diffusion, is controlled by the mass transfer in the matrix itself. Particularly, diffusion-controlled systems are divided into monolithic (matrix) and reservoir (membrane or core-shell) controlled systems (14). In the first case, the drug is dissolved and uniformly distributed on a polymeric support. If its content is greater than the drug solubility in the polymer, only part of the agent is dissolved and the remainder is dispersed as fine solid particles. In the reservoir system, the drug and the barrier material (often polymers) which controls the release rate are physically separated; the drug is at the center of the dosage form, while the polymer forms a membrane that surrounds the drug reservoir (capsule).

At the macroscopic level, the diffusion of drug molecules through the polymer can be described by the Fick's law of diffusion. Based on this equation, several empirical and semiempirical models were developed. The Higuchi equation (15) 
is a famous and used mathematical approach to describe drug release rates from matrix systems. An important advantage of this equation is its simplicity. However, when applied, the assumptions of the Higuchi model should be considered: drug concentration is above its solubility threshold, drug diffusivity remains constant, and perfect sink conditions are maintained, among others.

A more comprehensive, but still very simple, semiempirical equation to describe drug release from polymeric systems is the so-called power law or Korsmeyer-Peppas equation (16).

$\frac{M_{t}}{M_{\infty}}=k t^{n}$

where $M_{t}$ and $M_{\infty}$ are the amounts of drug released at time $t$ and at equilibrium. The fractional amount of drug released $\left(M_{t} / M_{\infty}\right)$ at time $t$ is expressed as a constant $k$, incorporating structural and geometric characteristics of the device and a potential relationship with time, where $n$ is the release exponent, which varies between $0<n<1$ and provides information about the drug release mechanism. Therefore, depending on the $n$ value, two types of mechanism can be assumed: (a) for $0.4<n<0.5$ (depending on device geometry), a Fickian diffusion occurs, indicating diffusion-controlled drug release; (b) when $n \cong 1.0$, it indicates that transport mechanism involving swelling-controlled drug release can be assumed. Values of $0.5<n<1.0$ is an indicative of superposition of both phenomena (anomalous transport). This equation's main disadvantage is that the $n$ coefficient may take a value of 0.5 which would indicate a diffusion-controlled drug release mechanism, and this value may nevertheless be due to the superposition with other effects such as polymer swelling, degradation, or erosion, occurring during drug release (10). It should be noticed that (Eq. 1) could only be applied for a total amount of drug release lower than $60 \%$.

In the veterinary field, drug administration through controlled-release systems is very interesting because it reduces the number of interventions by the veterinarian, which means less stress for animals and lower costs. Moreover, they are important to achieve effective control in reproduction. In this context, progesterone is widely used in the control of estrogen. The controlled release of progesterone can maintain the proper level of drug used in insemination protocols, allowing ovulation synchronization. There are several studies that incorporate progesterone in different polymeric matrices with this purpose (17-22).

Among the materials used to prepare these systems, polymers are of interest, particularly those that are biodegradable and from natural sources. There is growing interest for the group of biopolymers known as polyhydroxyalkanoates, which are biodegradable and biocompatible linear polyesters that have proven to be excellent candidates for medical and pharmaceutical applications (23-30).

Particular attention has been focused on the use of poly(3hydroxybutyrate) (PHB). This polymer is synthesized by bacterial fermentation and, unlike other members of polyhydroxyalkanoates family (polyhydroxyvalerate, polyhydroxyhexanoate), is produced in greater amount by bacteria of different genera (31). This polymer is biocompatible, biodegradable, and can be converted completely into carbon dioxide and water by the action of microorganisms in natural environments $(32,33)$. For these reasons, it has been widely studied for medical uses, food, and packing material as well (33-36).

In the present contribution, technological research is based on a case of special relevance for countries with large dairy and livestock production. It is focused on the administration of meat production promoters such as progesterone (Prg), using biodegradable polymeric membranes as platform for the drug-controlled release. The drug is incorporated into PHB matrices, evaluating the Prg effect on morphological and structural membrane properties.

We present a pre-formulation study concerning the design of films to be used in drug delivery systems in the mucosa. The management of estrous control of livestock through pharmacologic intervention using intravaginal inserts containing progesterone represents a valuable farm management tool to enhance production, enrich genetic stock characteristics, and so forth (37). The vagina of livestock animals, such as cattle and sheep, is an attractive site for drug delivery because of the easiness with which drugs are administered, the easiness to remove the system as well (if it is not biodegradable), and its ability to retain drug delivery systems for prolonged periods (weeks) (37). Intravaginal veterinary drug delivery systems have been studied and developed for the administration of synthetic and natural hormones such as progesterone, methyl acetoxy progesterone, fluorogestone acetate, and estradiol benzoate (38-40).

The availability of new materials and the development of innovative delivery systems broaden the research field to reach successful platforms.

The objectives of this work were to design, characterize and assess the progesterone release from these innovative systems using engineering approaches that were extrapolated to the pharmaceutical field, by our research group. Transport and kinetic phenomena involved in drug release processes were both considered by the lumped model. Furthermore, these models comply with the declaration done 40 years ago by Aris (41) and more recently by Levenspiel (42): "models are needed as simple as possible to provide a good description of the system behavior." Release phenomena and their connection to the structural properties of the synthesized membranes were established.

\section{EXPERIMENTAL}

\section{Materials}

The polymer PHB was used (Fig. 1a). Powder PHB $(\mathrm{Mw} \approx 524,000 \mathrm{~g} / \mathrm{mol})$ was kindly provided by BIOCYCLE®, PHB Industrial S.A. (Brazil) with a purity of $99.5 \%$ and moisture content below $0.3 \%$. Chloroform, by Cicarelli (Argentina), was used as solvent and Prg as drug (Farmabase, Rovereto, Italy) (Fig. 1b). All chemicals were of analytical grade and used without further purification.

\section{Membrane Synthesis}

Membranes were prepared by the solution-casting technique (43). PHB was dissolved in chloroform at $60^{\circ} \mathrm{C}$ for $4 \mathrm{~h}$ under reflux, to keep the concentration constant. Membrane samples were prepared by casting the chloroform solution in 


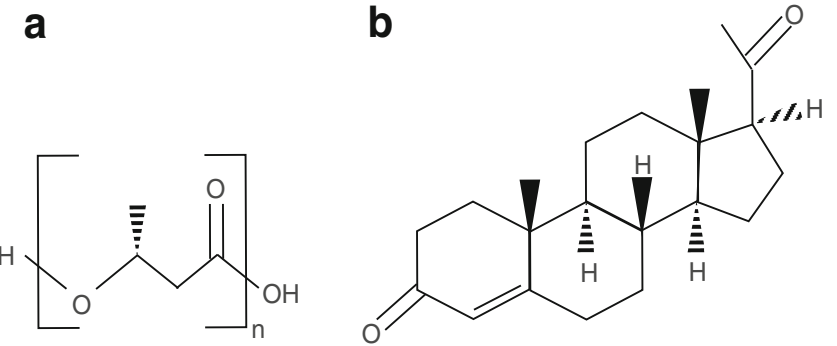

Fig. 1. Chemical structure of a poly(3-hydroxybutyrate) (PHB) and b progesterone (Prg). Above and below-the-plane bonds indicate methyl groups $\left(\mathrm{CH}_{3}\right)$.

glass Petri dishes. Solvent evaporation was completed after $24 \mathrm{~h}$ at room temperature. For progesterone-loaded membranes, the drug was introduced by direct dispersion in the polymeric solution, stirring constantly at room temperature. Finally, the films were obtained using the same procedure for pure polymeric membranes. Drug content ranged from 23 to $33 \mathrm{wt} \%$. Once the solvent evaporation process was completed, an IR spectroscopy study was performed in order to verify the absence of chloroform in the loaded membranes.

Membrane thicknesses were measured by an Electronic Outside Micrometer 0-1", 54-850-001 (Fowler, China) and verified with cross section membranes scanning electron microscopy (SEM) images (around $110 \mu \mathrm{m}$ ).

\section{Characterization}

The melting temperatures of Prg, composite membranes (PHB - progesterone), and physical mixture of both components were determined using differential scanning calorimetry, DSC882E-Mettler Toledo, from the Bioforge Institute, Valladolid University, Spain. Each sample was sealed in an aluminum pan, and all scans were carried out under nitrogen atmosphere $\left(22.5 \mathrm{ml} / \mathrm{min}^{-1}\right)$. The samples were heated from $-20^{\circ} \mathrm{C}$ to $200^{\circ} \mathrm{C}$ at a heating rate of $10^{\circ} \mathrm{C} / \mathrm{min}$.

Cross sections of the membranes have been observed by SEM (JEOL JSM-6480 LV), LASEM laboratory (UNSa - CONICET). The dried samples were fractured in liquid nitrogen, to avoid structure deformations, sputtered with a thin layer of gold (Denton Vacuum LLC Desk IV Sputter), and then mounted on the sample stand.

Fourier transform infrared spectroscopy (FTIR) data were recorded on a GX FTIR Perkin Elmer Spectrometer. Transmission spectra of powder PHB and Prg were obtained from the samples diluted with KBr. For PHB-Prg membranes, IR was performed by transmission with the membranes casted over silicon wafers.

\section{In Vitro Release Experiments}

Experimental procedures are of crucial importance to know exactly what the data obtained means and how it must be treated to fit mathematical model or to compare results that come using different experimental techniques.

The in vitro release data measurements were performed using a pre-weighed progesterone-loaded membrane, of a known thickness, placed in contact with the release medium ( $\mathrm{pH} 6.8$ buffer phosphate solution), at $32^{\circ} \mathrm{C}$. Samples were withdrawn at preset time intervals and the amount of progesterone released was determined in triplicate using UV-visible spectroscopy (UV-Visible 2100C) at $245 \mathrm{~nm}$. The validation of the analytical method was performed as indicated in the ICH guideline (44). The hormone releases from the membranes were performed under two different techniques:

- Technique A, constant volume of the release medium: the Prg-loaded membrane was brought into contact with $100 \mathrm{ml}$ of the release medium. A $3 \mathrm{ml}$ sample volume was extracted for UV spectrophotometry quantification and then immediately returned to the original solution, to maintain the total volume constant (it was not replaced by equal volume of fresh medium). This procedure fulfills the batch process conditions (i.e., constant mass of drug in the system). Samples were taken every $15 \mathrm{~min}$ in the first hour, and then every $30 \mathrm{~min}$. Each experiment lasts at least $4 \mathrm{~h}$.

- Technique B, total extraction volume was withdrawn: drugloaded membrane was introduced in $10 \mathrm{ml}$ solution. For UV spectroscopy quantification, the entire release medium was removed and replaced with an equal volume of fresh medium. This technique follows "perfect sink conditions" (i.e., the drug concentration in the surrounding fluid is negligible at all times) (13). This procedure was repeated every $15 \mathrm{~min}$ during the first hour, and then every $30 \mathrm{~min}$. Each experiment lasts at least $4 \mathrm{~h}$.

With these procedures, if the membrane is kept in solution long enough, the amount released can be experimentally well quantified, but the change is relatively small to be considered differential; then the drug released rate can be expressed as follows:

$\frac{d M_{t}}{d t} \cong \frac{\Delta\left(M_{t}\right)}{\Delta t}$

$M_{t}$ is the cumulative amount of drug released at time $t$. Thus, when technique $\mathrm{B}$ was used, the highest drug release rate at each time was found, since the driving force was always the maximum one.

The progesterone loads employed in both experimental techniques were 23,29 , and $33 \mathrm{wt} \%$. The films were fixed with a metal mesh and then placed at the bottom of the vessel for technique A (membranes of $3 \times 4 \mathrm{~cm}$ ) and in tubes for technique $B$ (membranes of $1 \times 3 \mathrm{~cm}$ ). Vessel and tubes were set in a continuous moving shaker bath (45-47). For each composition and technique, three membrane samples were evaluated and the mean value was reported.

\section{Modeling of Release Profiles}

In general, the mechanisms involved in the drug control release consider several steps. These steps can be as follows: drug dissolution, diffusion through the polymeric matrix, eventually polymer swelling, and then transference to the receptor solution at the membrane-fluid interface. The set of differential equations that describe these proesses are complex and needs to be solved numerically. Therefore, a single explicit analytical solution function between the mass of drug release and time are not possible to obtain.

We propose a mathematical model that follows a lumped second-order kinetics. The drug release rate is directly 
proportional to the square of the amount of drug available in the membrane at each moment (Eq. 3). We found that this model satisfactorily describes processes where diffusion and transfer phenomena are present, or when only external transfer exists into a fluid medium where the drug concentration increases steadily (48-51).

$\frac{\mathrm{d} M_{t}}{\mathrm{~d} t}=k_{1}\left(M_{\infty}-M_{t}\right)^{2}$

$M_{t}$ is the total amount of drug released (technique A), or the cumulative mass of drug released (technique B), at time $t$, respectively. $M_{\infty}$ is the total amount of drug feasible to be released at equilibrium. By elementary integration of this differential equation, between the initial condition $(t=0$, $\left.M_{t}=0\right)$ and any other $\left(t=t, M_{t}=M_{t}\right)$, Eq. 4 is obtained:

$M_{t}=\frac{M_{\infty}^{2} \times k_{1} \times t}{\left[1+\left(M_{\infty} \times k_{1}\right) \times t\right]}=\frac{a \times t}{[1+b \times t]}$

Where

$a=M_{\infty}^{2} \times k_{1}$ and $b=M_{\infty} \times k_{1}$

Therefore,

$\frac{a}{b}=M_{\infty}$

Results obtained with both techniques fit this model.

Eq. 4 can be put in linear forms as follows:

$\frac{t}{M_{t}}=\frac{1}{a}+\left(\frac{b}{a}\right) \times t$

By using Eq. 7 and making the graphic of $\left(t / M_{t}\right)$ versus time $(t)$, the values of parameters $a$ and $b$ are obtained.

However, the best procedure to fit the model with experimental data is to carry out a nonlinear regression analysis using as first guess the values of $a$ and $b$ found graphically. We did the nonlinear regression analysis applying the Polymath 5.1 program.

\section{RESULTS AND DISCUSSION}

\section{Scanning Electron Microscopy}

Figure 2 shows SEM images of powder Prg (Fig. 2a), pure PHB membrane (Fig. 2b), and a PHB membrane loaded with $33 \mathrm{wt} \%$ of Prg (Fig. 2c). SEM images showed that the PHB membrane structure is fully dense, which remains after incorporating the drug. For the PHB-Prg membranes, the presence of progesterone crystals was observed, suggesting that their content is above the solubility limit in the polymer. This is of importance in modeling the drug release kinetics, the assumptions made, and the boundary conditions assumed. The distribution of the crystals was uniform through the entire thickness of the membranes, indicating that the drug incorporation in the film was effective.

\section{Differential Scanning Calorimetry}

Differential scanning calorimetry (DSC) was used to determine the melting temperature of Prg, PHB-Prg powder physical mixtures, and Prg-loaded PHB membranes. Pure PHB has two melting temperatures, a common behavior in several semicrystalline polymers and was previously reported by one of the authors (52).

Progesterone melting point is $131^{\circ} \mathrm{C}$. When it is physically mixed with powder $\mathrm{PHB}$, this value is maintained while $\mathrm{PHB}$ melting point is $170^{\circ} \mathrm{C}$ (Fig. 3). Melting temperature values decrease slightly as the progesterone is incorporated in the PHB matrix $\left(T_{\mathrm{Prg}}=118^{\circ} \mathrm{C}\right.$ and $\left.T_{\mathrm{PHB}}=160^{\circ} \mathrm{C}\right)$ (Fig. 3). The reductions of melting temperatures observed in the case of polymeric blends are associated to the weakening of polymer-polymer interactions and changes in the polymer structure (17). So in this case, progesterone has a plasticizer effect within the PHB polymer chains. The Prg melting peak in the membrane is broader than that for the pure drug, confirming the existence of polymer chain-drug interactions (53).

\section{FTIR Spectroscopy}

The FTIR spectra of pure Prg, PHB, and PHB-Prg membranes with different therapeutic agent loadings are shown in Fig. 4. In the spectrum of pure progesterone (Fig. 4(a)), there are two sharp and well-resolved bands at $1662 \mathrm{~cm}^{-1}\left(v_{1}\right.$ $(\mathrm{C}=\mathrm{O}))$ and $1698 \mathrm{~cm}^{-1}\left(v_{2}(\mathrm{C}=\mathrm{O})\right)$ assigned to carbonylstretching bands of $\mathrm{C}_{3}$ and $\mathrm{C}_{20}$, respectively, and a lessintensive band at $1615 \mathrm{~cm}^{-1}$ corresponding to the stretching vibration band of $\mathrm{C}=\mathrm{C}(54-56)$.

The pure PHB spectra (Fig. 4(b)) can be divided in the following areas: the $3020-2830 \mathrm{~cm}^{-1}$ range (the bands correspond to $\mathrm{C}-\mathrm{H}$ stretching vibration), the peak in $1727 \mathrm{~cm}^{-1}$ (the most intensive band due to $\mathrm{C}=\mathrm{O}$ stretching vibration of the crystalline ester carbonyl group), and the $1500-800 \mathrm{~cm}^{-1}$ range (due to the $\mathrm{CH}_{3}, \mathrm{CH}_{2}, \mathrm{CH}$ bending vibration, $\mathrm{C}-\mathrm{O}-\mathrm{C}$ and $\mathrm{C}-\mathrm{C}$ stretching vibration (57)). The peak in $1057 \mathrm{~cm}^{-1}$ is assigned to $\mathrm{C}-\mathrm{O}$ stretching vibrations from alcohols. The peaks in 1185 and $1280 \mathrm{~cm}^{-1}$ are assigned to the stretching vibrations of the ester C$\mathrm{O}-\mathrm{C}$ group. The peak in $1229 \mathrm{~cm}^{-1}$ is due to the $\mathrm{CH}_{2}$ wagging and twisting modes (58).

The FTIR spectrum of PHB-Prg-loaded membranes shows the characteristic bands of Prg. The band at $1698 \mathrm{~cm}^{-1}$ is overlapped with the intensive PHB band at $1727 \mathrm{~cm}^{-1}$. The peak at $1662 \mathrm{~cm}^{-1}$ appears shifted to $1669 \mathrm{~cm}^{-1}$, which could be attributed to a weak hydrogen bonding (22). The band intensity increases with the Prg content in membranes.

Moreover, as stated in "Membrane synthesis", FTIR studies were carried out in order to verify complete chloroform evaporation from the membranes. Chloroform has the most intense IR bands at $3020 \mathrm{~cm}^{-1}(\mathrm{C}-\mathrm{H}$ stretching vibrations), $1219 \mathrm{~cm}^{-1}$ (C-H bends), $773 \mathrm{~cm}^{-1}$ (C-Cl stretching vibrations), and $671 \mathrm{~cm}^{-1}$ (C-Cl bends). The first band mentioned $\left(3020 \mathrm{~cm}^{-1}\right)$ was used for monitoring traces of solvent. This band is placed at higher frequencies than those due to CH PHB vibrations, whose main peaks appear at 2975 and $2934 \mathrm{~cm}^{-1}$ (Fig. 4).

Furthermore, the band at $1219 \mathrm{~cm}^{-1}$ is not appropriate for chloroform detection due to the overlap with PHB bands. Finally, no bands were observed at lower frequencies (800- 

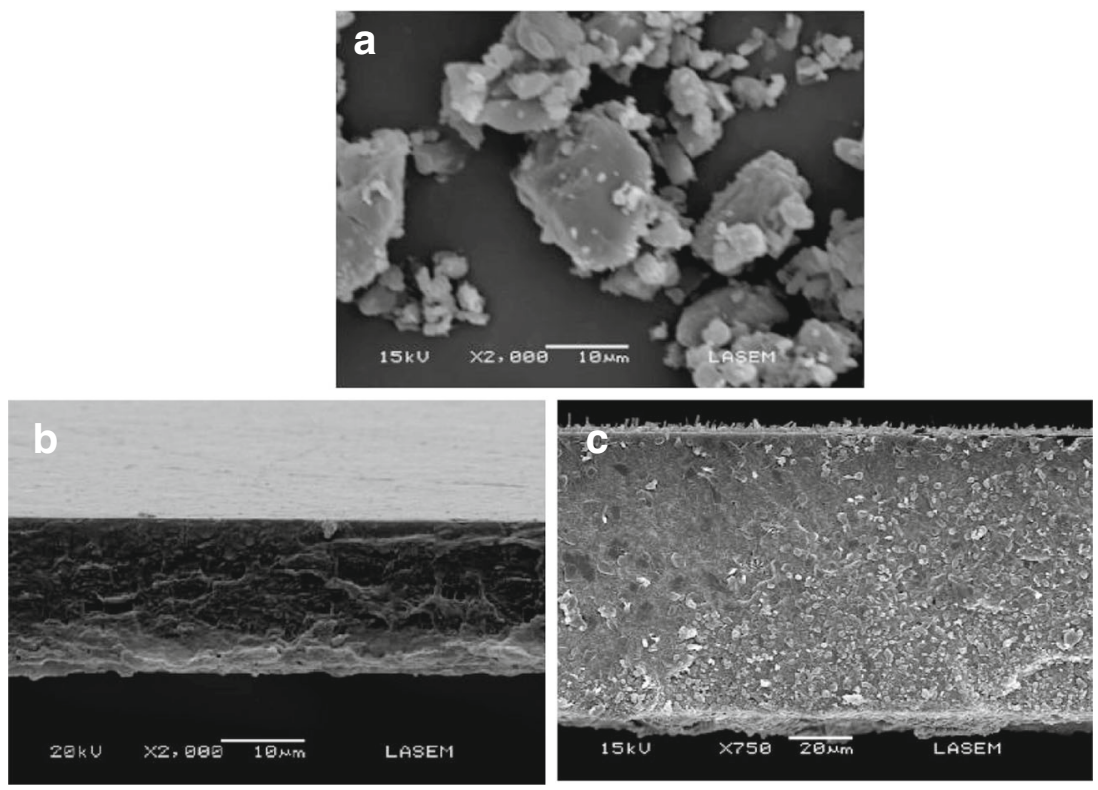

Fig. 2. SEM micrographs of a powder Prg, b pure PHB cross section membrane, and $\mathbf{c}$ Prgloaded PHB membrane: PHB-Prg, 33 wt $\%$

$650 \mathrm{~cm}^{-1}$ range) in loaded membrane spectra (not shown in the figure), confirming solvent absence.

\section{In Vitro Progesterone Release: Modeling}

\section{Technique A: Returning the Sample to the Original Solution}

Figure 5 shows the progesterone mass release from PHBPrg membranes, with different Prg content, as function of

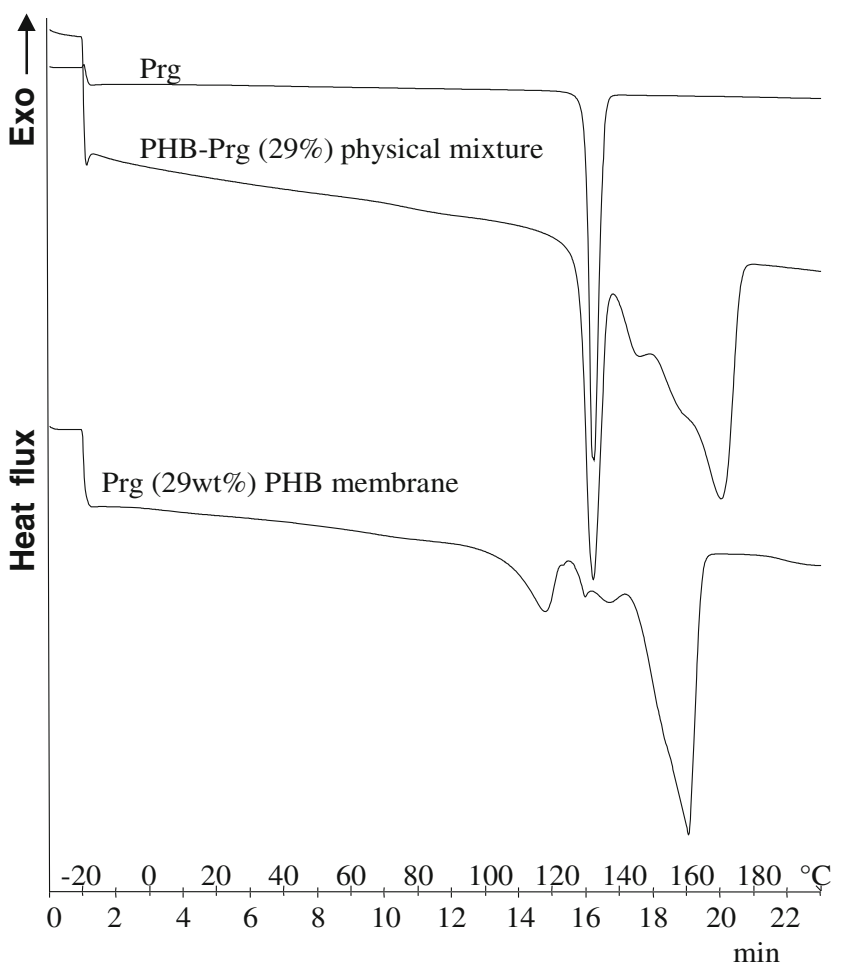

Fig. 3. DSC of progesterone, PHB-Prg (29 wt $\%$ ) physical mixture, and $\operatorname{Prg}(29 \mathrm{wt} \%)$-loaded PHB membranes time. It can be seen that the amount of hormone released is influenced by the original progesterone loaded in the membrane; the higher the Prg load, the lower the drug release. This behavior can be attributed to the presence of an excess of progesterone crystals in the membrane and particularly at the interface. Dissolution of these crystals in the release medium is hindered by the very low Prg solubility in the polymer as well in the receptor solution. Thus, the effective surface available for drug release decreases as Prg loading increases. When the dissolved and non-dissolved Prg coexist within the polymeric matrix, the dissolved drug is the only available for diffusion.

Chang et al. (18) found for the progesterone/poly $(\varepsilon-$ caprolactone) (PCL) system that the cumulative released amount was higher for the samples with lower drug content. Progesterone loads in PCL matrices influenced the amount of drug released and the time at which the process is carried out. However, the pattern of drug release was similar among the different loads they studied. The authors argue that progesterone delivery may be controlled by factors influencing drug dissolution (such as the dispersed particles sizes and solubility) rather than diffusion. Benita et al. (59) incorporated nifedipine as model drug in polyacrylate, a poorly water-soluble drug as progesterone. They reported that an increase in drug concentration decreased the release rate due to drug crystalline domains formation in the microspheres. They also concluded that nifedipine dissolution in the microspheres controls the overall release kinetic process since it is probably the slowest step in the kinetic process.

Experimental data showed a very good fit through a nonlinear regression analysis (Polymath 5.1 program) (Fig. 5). Thus, $a$ and $b$ parameters were determined, and $M_{\infty}$ and $k_{1}$ values were calculated (Eqs. 5-6) and reported in Table I with a $95 \%$ confidence level.

Note that the model can be applied for $0 \leq t \leq \infty$, since for $t \rightarrow \infty$, the model gives $M_{\infty}$ (6). 


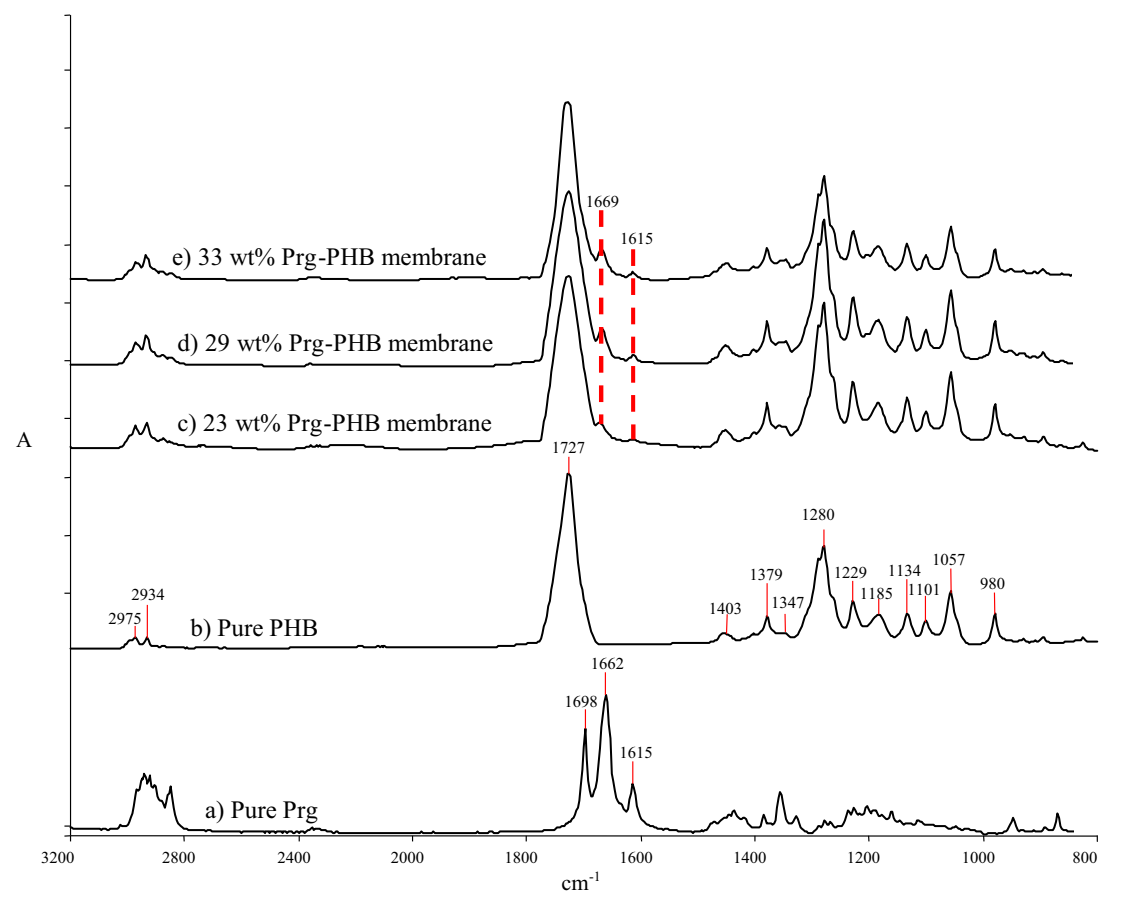

Fig. 4. Infrared spectra of $a$ pure Prg; $b$ pure PHB membrane; and Prg-PHB membranes with $c 23 \mathrm{wt} \% \operatorname{Prg}, d 29 \mathrm{wt} \% \operatorname{Prg}$, and $e 33 \mathrm{wt} \% \operatorname{Prg}$

The release rates are obtained deriving $M_{t}$ versus time in Eq. 4, which results in Eq. 8.

$\frac{\mathrm{d} M_{t}}{\mathrm{~d} t}=\frac{a}{(1+b \times t)^{2}}$

Technique B: Release Assays with Complete Removal of the Medium

The total mass of released Prg showed a linear increase with time (Fig. 6), attributable to a constant driving force due to the high concentration of progesterone in the membrane, i.e., constant membrane-fluid surface concentration over time, as well as the use of the same volume of fresh solution $\left(M_{t} \approx 0\right)$, resulting in a simple external mass transfer process. Therefore Eq. 3 reduces to

$\frac{\mathrm{d} M_{t}}{\mathrm{~d} t}=k_{1} \times M_{\infty}^{2}=k_{2}$

After integration, the linear model represented by Eq. 10 is obtained.

$M_{t}=k_{2} \times t$

The cumulative drug release profiles performed under technique $\mathrm{B}$, in this contribution (total volume extraction), follows Eq. 10.

Regarding relationship between the Prg released and the initial amount in the membrane, the same behavior as technique $\mathrm{A}$ (when the sample was returned to the original solution) was observed. At higher initial Prg load, drug release rate lowers. Dotted lines in Fig. 6 correspond to Eq. 10; $k_{2}$ and $R^{2}$ values, obtained by regression analysis, are presented in Table II with a $95 \%$ confidence level. In this case, the drug release rate was constant as predicted by Eq. 9.

Finally, comparative analysis of release rates obtained by both drug extraction techniques was carried out. The release rates were normalized considering both the effective membrane interface area and the extractor liquid volume. At the beginning of the release process $(t=0)$, the system conditions were the same for both techniques. Therefore, the normalized release rates $(N R R)$ should be equal for both experimental procedures used.

Due to the low Prg solubility in the PHB, the density of both PHB and Prg could be considered as pure compounds, i.e.,

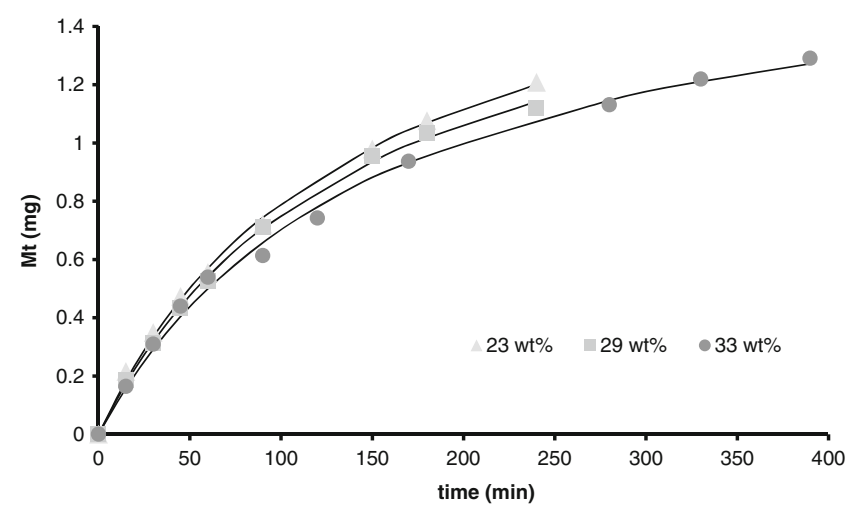

Fig. 5. Prg concentration effect on the drug release profile from the PHB-Prg membranes using technique A. Symbols are the mean value experimental data and their sizes represent the standard deviation. Lines represent the theoretical release predictions with nonlinear regression fit developed in this work (Eq. 4) 
Table I. Coefficients of Model: $M_{t}=a \times t /(1+b \times t)($ Eq. 4$)$

\begin{tabular}{lccc}
\hline Parameters & \multicolumn{3}{l}{ Progesterone wt\% } \\
\cline { 2 - 4 } & 23 & 29 & 33 \\
\hline$a(\mathrm{mg} / \mathrm{min})$ & $1.36 \times 10^{-2} \pm$ & $1.28 \times 10^{-2} \pm$ & $1.16 \times 10^{-2} \pm$ \\
& $1.04 \times 10^{-3}$ & $7.10 \times 10^{-6}$ & $1.20 \times 10^{-3}$ \\
$b\left(\mathrm{~min}^{-1}\right)$ & $7.14 \times 10^{-3} \pm$ & $7.08 \times 10^{-3} \pm$ & $6.58 \times 10^{-3} \pm$ \\
& $1.04 \times 10^{-3}$ & $7.50 \times 10^{-6}$ & $1.60 \times 10^{-3}$ \\
$R^{2}$ & 0.998 & 0.999 & 0.998 \\
$M_{\infty}(\mathrm{mg})$ & $1.90 \pm 3.50 \times 10^{-2}$ & $1.81 \pm 1.80 \times 10^{-2}$ & $1.77 \pm 3.60 \times 10^{-2}$ \\
$k_{1}(\mathrm{mg} / \mathrm{min})$ & $3.75 \times 10^{-3} \pm$ & $3.90 \times 10^{-3} \pm$ & $3.72 \times 10^{-3} \pm$ \\
& $1.00 \times 10^{-4}$ & $7.20 \times 10^{-5}$ & $1.80 \times 10^{-4}$ \\
\hline
\end{tabular}

$1.166 \mathrm{~g} / \mathrm{cm}^{3}$ for Prg and $1.300 \mathrm{~g} / \mathrm{cm}^{3}$ for PHB. Therefore, the volume fraction of PHB for the three Prg-loaded membranes could be obtained. These values are $0.750,0.687$, and $0.645 v / v$ PHB for the 23, 29, and $33 \mathrm{wt} \%$ Prg, respectively.

Considering the stereometric principle, the PHB volume fraction of the membrane is equal to the fraction of area exposed (due to the uniform distribution of Prg crystal in the membrane, Fig. 2c). Then, the "effective" area $\left(A_{\text {ef }}\right)$ exposed by the membrane is the total interface area of each membrane multiplied by the volume fraction (surface fraction) of PHB. The normalized release rate using techniques $\mathrm{A}\left(N R R_{A}\right)$ and $\mathrm{B}\left(N R R_{B}\right)$ are expressed in Eqs. 11 and 12, respectively $\left(\mathrm{mg} \mathrm{cm}_{\mathrm{ef}}^{2} / \mathrm{min} / \mathrm{ml}\right)$.

$$
\begin{aligned}
& N R R_{\mathrm{A}}=\frac{a}{A_{\mathrm{ef}} V_{\mathrm{A}}} \\
& N R R_{\mathrm{B}}=\frac{k_{2}}{A_{\mathrm{ef}} V_{\mathrm{B}}}
\end{aligned}
$$

where $V_{\mathrm{A}}$ and $V_{\mathrm{B}}$ are the release medium volumes used for techniques $\mathrm{A}$ and $\mathrm{B}, 100$ and $10 \mathrm{ml}$, respectively. Results are reported in Table III.

For both techniques the $N R R$ values at $t=0$ were approximately equal, with a mean value of $7.26 \times 10^{-6} \pm 8 \%$,

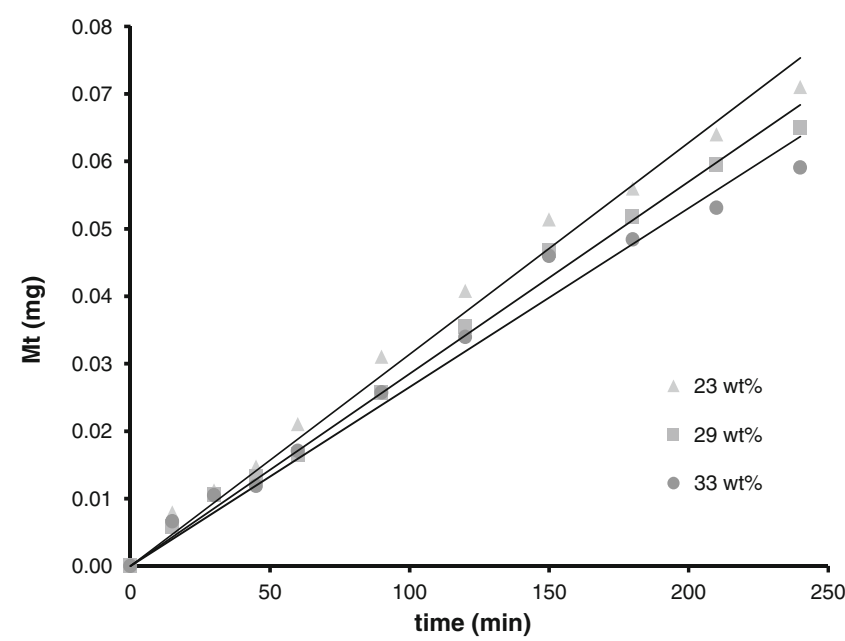

Fig. 6. Prg concentration effect on the drug release profile from the PHB-Prg membranes using technique B. Symbols are the mean value experimental data and their sizes represent the standard deviation. Lines represent the theoretical drug release predictions with our model
Table II. Coefficient of Model: $M_{t}=k_{2} \times t$ (Eq. 10)

\begin{tabular}{llcc}
\hline Parameters & \multicolumn{3}{l}{ Progesterone wt\% } \\
\cline { 2 - 4 } & 23 & 29 & 33 \\
\hline$k_{2}(\mathrm{mg} / \mathrm{min})$ & $3.14 \times 10^{-4} \pm$ & $2.85 \times 10^{-4} \pm$ & $2.65 \times 10^{-4} \pm$ \\
& $1.40 \times 10^{-5}$ & $9.80 \times 10^{-6}$ & $1.50 \times 10^{-5}$ \\
$R^{2}$ & 0.987 & 0.993 & 0.979 \\
\hline
\end{tabular}

considering experimental error and model fitting, for the three Prg-loaded membranes. This supports the experimental results, showing internal consistency of the experimental methodology and the model proposed.

Another evidence that the membrane effective area is the PHB surface area fraction are the values calculated for the release rate per unit of effective surface area (RRef) $\left(\mathrm{mg} \mathrm{cm}_{\mathrm{ef}}^{2} / \mathrm{min}\right)$ in the complete time interval. For example, when technique A was used (see Eq. 8), RRef is calculated with Eq. 13.

$R \operatorname{Ref}=\frac{a^{\prime}}{(1+b \times t)^{2}}$

with

$a^{\prime}=\frac{a}{A_{\mathrm{ef}}}$

For technique A, the values of $a^{\prime}$ are $7.54 \times 10^{-4}, 7.78 \times 10^{-4}$, and $7.51 \times 10^{-4}$, for the 23,29 , and $33 \mathrm{wt} \%$ Prg membranes.

Figure 7 shows that a single RRef curve fits the results for the three cases in the complete time range. The RRef is maximum at the beginning of the release experiments, decreasing to very low values after $4 \mathrm{~h}$ of contact of the membrane with the medium, as expected.

Regarding the progesterone dissolved in the membrane, an indirect prediction of the Prg solubility in PHB can be done using the obtained data with technique A. Taken into account the membrane volume $\left(V_{\mathrm{m}}\right)$, the volume fraction of PHB $(v /$ $v \mathrm{PHB})$ and $M_{\infty}$ values found for each membrane, the Prg solubility in PHB $\left(C_{p r g}^{\circ}\right)$ is estimated as

$C_{P r g}^{\mathrm{o}}=\frac{M_{\infty}}{\left(V_{\mathrm{m}}\right) \times(v / v \mathrm{PHB})}$

The mean value obtained is $\left(C_{p r g}^{\circ}\right)=20.00 \pm 0.52 \mathrm{mg} /$ $\mathrm{cm}^{3}$ PHB.

Table III. Normalized Release Rates $(N R R)\left(\mathrm{mg} / \mathrm{cm}_{\mathrm{ef}}{ }^{-2} / \mathrm{min} / \mathrm{ml}\right)$, at

\begin{tabular}{|c|c|c|c|}
\hline & \multicolumn{3}{|c|}{ Progesterone load } \\
\hline & $\begin{array}{l}23 \mathrm{wt} \% \\
(0.75 v / v \mathrm{PHB})\end{array}$ & $\begin{array}{l}29 \mathrm{wt} \% \\
(0.687 v / v \mathrm{PHB})\end{array}$ & $\begin{array}{l}33 \mathrm{wt} \% \\
(0.645 v / v \text { PHB })\end{array}$ \\
\hline$N R R_{A}$ & $7.54 \times 10^{-6}$ & $7.78 \times 10^{-6}$ & $7.51 \times 10^{-6}$ \\
\hline$N R R_{B}$ & $7.16 \times 10^{-6}$ & $6.91 \times 10^{-6}$ & $6.85 \times 10^{-6}$ \\
\hline
\end{tabular}
$t=0$

$N R R_{A}$ normalized release rate using technique $\mathrm{A}, N R R_{B}$ normalized release rate using technique $\mathrm{B}, v / v P H B$ volume fraction of $\mathrm{PHB}$ 


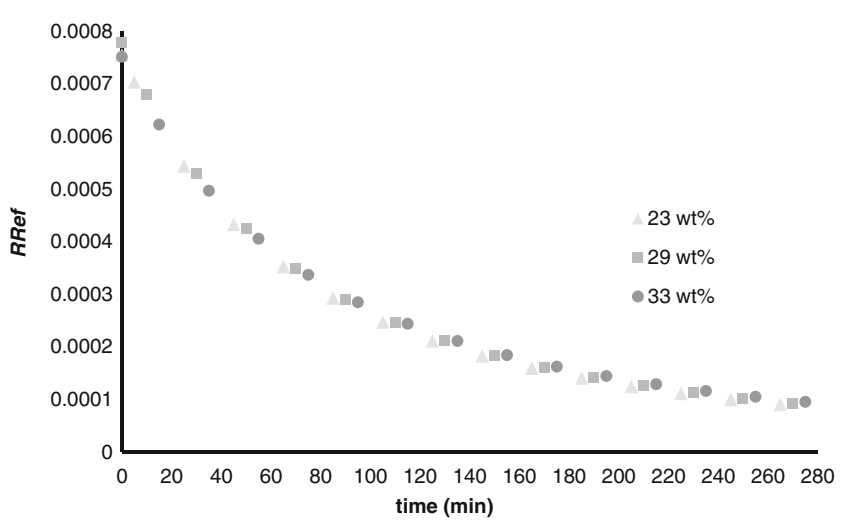

Fig. 7. RRef vs. time for technique A (Eq. 13)

The solubility of the progesterone in the release medium was $0.021 \pm 0.001 \mathrm{mg} / \mathrm{ml}$, measured according to the procedure indicated by Grant and Higuchi (60).

\section{CONCLUSIONS}

Results showed that PHB films are able to control progesterone release, which would reduce the variability in drug performance. The latter aspect is increasingly important given the current emphasis on pharmaceutical quality by design (QbD) by regulatory agencies such as the Food and Drug Administration (FDA).

Result analysis using our proposed approach yielded simple and explicit relations between mass and drug released rate. The model can be applied without limitations of time nor mass of drug released. The kinetic parameters determined could be extrapolated to in vivo findings and could qualitatively provide insight into the potential use of these systems in veterinary.

Internal consistency of both experimental procedures used in this work was verified. Furthermore, a single RRef for the three loaded membranes was found, which confirms that the PHB fraction in the membrane interface is the effective surface for the mass transference.

\section{ACKNOWLEDGMENTS}

The authors would like to thank CONICET (Argentina) for research fellowships, CIUNSa (Grant 1469/0, 1471, and 1895), CONICET (PIP $11220120100313 C O$ ), ANPCyT (PICT 2012 - 2643), and ANPCyT- MICINN (Argentina-Spain) (PICT 2011-2751). They also thank BIOCYCLE®, PHB Industrial S.A. (Brazil) for providing the polymer, Eng. Silvia Blanco from LASEM laboratory, ANPCyT - UNSa CONICET, and Prof. Lilian Davies from FTIR laboratory, for their willingness, and Bioforge Institute, Valladolid University, Spain, for DSC measurements.

\section{REFERENCES}

1. Siepmann J, Peppas NA. Modeling of drug release from delivery systems based on hydroxypropyl methylcellulose (HPMC). Adv Drug Deliv Rev. 2012;64(0):163-74.

2. Siepmann J, Kranz H, Bodmeier R, Peppas NA. HPMCmatrices for controlled drug delivery: a new model combining diffusion, swelling, and dissolution mechanisms and predicting the release kinetics. Pharm Res. 1999;16((11):1748-56.

3. Fu Y, Kao WJ. Drug release kinetics and transport mechanisms of non-degradable and degradable polymeric delivery systems. Exp Opin Drug Deliv. 2010;7(4):429-44.

4. Pham A, Lee P. Probing the mechanisms of drug release from hydroxypropylmethyl cellulose matrices. Pharm Res. 1994;11(10):1379-84.

5. Varma MS, Kaushal A, Garg A, Garg S. Factors affecting mechanism and kinetics of drug release from matrix-based oral controlled drug delivery systems. Am J Drug Deliv. 2004;2(1):43-57.

6. Langer R, Peppas N. Chemical and physical structure of polymers as carriers for controlled release of bioactive agents: a review. J Macromol Sci Part C: Polym Rev. 1983;23(1):61-126. doi:10.1080/07366578308079439.

7. Narasimhan B, Mallapragada SK, Peppas NA. Encyclopedia of controlled drug delivery. Mathiowitz E, editor. New York: Wiley; 1999.

8. Narasimhan B, Peppas NA. In: Park K, editor. Controlled drug delivery: challenges and strategies. Washington, DC: ACS; 1997.

9. Siepmann J, Peppas NA. Higuchi equation: derivation, applications, use and misuse. Int J Pharm. 2011;418(1):6-12.

10. Siepmann J, Siepmann F. Modeling of diffusion controlled drug delivery. J Contr Release. 2012;161(2):351-62.

11. Hennink WE, Franssen O, van Dijk-Wolthuis WNE, Talsma H. Dextran hydrogels for the controlled release of proteins. J Control Release. 1997;48(2-3):107-14.

12. Vergnaud J-M, Rosca I-D. Assessing bioavailability of drug delivery systems: mathematical modeling: CRC Press; 2005.

13. Siepmann J, Siegel RA, Rathbone MJ. Chapter 6-Diffusion controlled drug delivery systems. Fundamentals and applications of controlled release drug delivery

14. Stamatialis DF, Papenburg BJ, Gironés M, Saiful S, Bettahalli SNM, Schmitmeier S, et al. Medical applications of membranes: drug delivery, artificial organs and tissue engineering. J Memb Sci. 2008;308(1-2):1-34.

15. Higuchi T. Rate of release of medicaments from ointment bases containing drugs in suspension. J Pharm Sci. 1961;50(10):874-5.

16. Korsmeyer RW, Gurny R, Doelker E, Buri P, Peppas NA. Mechanisms of solute release from porous hydrophilic polymers. Int $\mathrm{J}$ Pharm. 1983;15(1):25-35.

17. Oliveira JE, Medeiros ES, Cardozo L, Voll F, Madureira EH, Mattoso LHC, et al. Development of poly(lactic acid) nanostructured membranes for the controlled delivery of progesterone to livestock animals. Mater Sci Eng C. 2013;33(2):8449.

18. Chang HI, Williamson MR, Perrie Y, Coombes AGA. Precipitation casting of drug-loaded microporous PCL matrices: Incorporation of progesterone by co-dissolution. J Control Release. 2005;106(3):263-72.

19. Jameela SR, Kumary TV, Lal AV, Jayakrishnan A. Progesterone-loaded chitosan microspheres: a long acting biodegradable controlled delivery system. J Control Release. 1998;52(1-2):17-24.

20. Matsumoto J, Nakada Y, Sakurai K, Nakamura T, Takahashi Y. Preparation of nanoparticles consisted of poly(l-lactide)-poly(ethylene glycol)-poly(l-lactide) and their evaluation in vitro. Int J Pharm. 1999;185(1):93-101.

21. Orienti I, Bigucci F, Luppi B, Cerchiara T, Zuccari G, Giunchedi $\mathrm{P}$, et al. Polyvinylalcohol substituted with triethyleneglycolmonoethylether as a new material for preparation of solid dispersions of hydrophobic drugs. Eur J Pharm Biopharm. 2002;54(2):229-33.

22. Izumikawa S, Yoshioka S, Aso Y, Takeda Y. Preparation of poly(l-lactide) microspheres of different crystalline morphology and effect of crystalline morphology on drug release rate. J Control Release. 1991;15(2):133-40.

23. Shrivastav A, Kim H-Y, Kim Y-R. Advances in the applications of polyhydroxyalkanoate nanoparticles for novel drug delivery system. Biomed Res Int. 2013;2013:12.

24. Williams SF, Martin DP. Applications of polyhydroxyalkanoates (PHA) in medicine and pharmacy. Biopolymers Online: WileyVCH Verlag GmbH \& Co. KGaA; 2005.

25. Tian H, Tang Z, Zhuang X, Chen X, Jing X. Biodegradable synthetic polymers: preparation, functionalization and biomedical application. Prog Polym Sci. 2012;37(2):237-80. 
26. Pouton $\mathrm{CW}$, Akhtar S. Biosynthetic polyhydroxyalkanoates and their potential in drug delivery. Adv Drug Deliv Rev. 1996;18(2):133-62.

27. Niaounakis M. Biopolymers: reuse, recycling, and disposal. USA: William Andrew Publishing; 2013.

28. Chen G-Q, Wu Q. The application of polyhydroxyalkanoates as tissue engineering materials. Biomaterials. 2005;26(33):6565-78.

29. Lobo F, Aguirre C, Silva M, Grillo R, Melo N, Oliveira L, et al. Poly(hydroxybutyrate-co-hydroxyvalerate) microspheres loaded with atrazine herbicide: screening of conditions for preparation, physico-chemical characterization, and in vitro release studies. Polym Bull. 2011;67(3):479-95.

30. Chen J, Davis SS. The release of diazepam from poly(hydroxybutyrate-hydroxyvalerate) microspheres. J Microencapsulation. 2002;19(2):191-201.

31. El-Hadi A, Schnabel R, Straube E, Müller G, Henning S. Correlation between degree of crystallinity, morphology, glass temperature, mechanical properties and biodegradation of poly (3hydroxyalkanoate) PHAs and their blends. Polym Test. 2002;21(6):665-74.

32. Zhang DM, Cui FZ, Luo ZS, Lin YB, Zhao K, Chen GQ. Wettability improvement of bacterial polyhydroxyalkanoates via ion implantation. Surface Coatings Technol. 2000;13(1):350-4. doi:10.1016/S0257-8972(00)00810-0.

33. Freier T, Kunze C, Nischan C, Kramer S, Sternberg K, Saß M, et al. In vitro and in vivo degradation studies for development of a biodegradable patch based on poly(3-hydroxybutyrate). Biomaterials. 2002;23(13):2649-57.

34. Siracusa V, Rocculi P, Romani S, Rosa MD. Biodegradable polymers for food packaging: a review. Trends Food Sci Technol. 2008;19(12):634-43.

35. Anderson AJ, Dawes EA. Occurrence, metabolism, metabolic role, and industrial uses of bacterial polyhydroxyalkanoates. Microbiol Rev. 1990;54(4):450-72.

36. Hasirci V. Biodegradable biomedical polymers. Review of degradation and in vivo responses to polylactides and polyhydroxyalkanoates. In: Wise DL, editor. Biomaterials and bioengineering handbook. New York: Marcel Dekker; 2000. p. $141-52$.

37. Rathbone MJ, Burns PJ, Ogle CR, Burggraaf S, Bunt CR. Chapter 7-Controlled release drug delivery systems for estrous control of domesticated livestock. In: Gurny MJR, editor. Controlled release veterinary drug delivery. Amsterdam: Elsevier; 2000. p. 201-28.

38. Rathbone MJ, Burke CR, Ogle CR, Bunt CR, Burggraaf S, Macmillan KL. Chapter 6-Design and development of controlled release intravaginal veterinary drug delivery systems. In: Gurny MJR, editor. Controlled release veterinary drug delivery. Amsterdam: Elsevier; 2000. p. 173200.

39. Rathbone MJ, Macmillan KL, Bunt CR, Shane B. Conceptual and commercially available intravaginal veterinary drug delivery systems. Adv Drug Deliv Rev. 1997;28(3):363-92.

40. Rathbone MJ, Foster TP. Veterinary pharmaceutical dosage forms. Modern pharmaceutics: applications and advances. 2009;2.

41. Aris R. Is sophistication really necessary? Ind Eng Chem. 1966;58(9):32-7. doi:10.1021/ie50681a008.
42. Levenspiel O. Modeling in chemical engineering. Chem Eng Sci. 2002;57(22-23):4691-6.

43. Mulder M, editor. Basic principles of membrane technology. Netherlands: Kluwer; 1991.

44. Guideline IHT. Validation of analytical procedures: text and methodology. Q2 (R1). 2005;1.

45. Llabot JM, Palma SD, Manzo RH, Allemandi DA. Design of novel antifungal mucoadhesive films. Part II. Formulation and in vitro biopharmaceutical evaluation. Int J Pharm. 2007;336(2):263-8.

46. Jelvehgari M, Mobaraki V, Montazam SH. Preparation and evaluation of mucoadhesive beads/discs of alginate and alginopectinate of piroxicam for colon-specific drug delivery via oral route. Jundishapur J Nat Pharm Prod. 2014;9(4):e16576.

47. Ahn J-S, Choi H-K, Chun M-K, Ryu J-M, Jung J-H, Kim Y-U, et al. Release of triamcinolone acetonide from mucoadhesive polymer composed of chitosan and poly(acrylic acid) in vitro. Biomaterials. 2002;23(6):1411-6.

48. Calleja G, Serna J, Rodríguez J. Kinetics of adsorption of phenolic compounds from wastewater onto activated carbon. Carbon. 1993;31(5):691-7.

49. Tseng R-L, Wu F-C, Juang R-S. Liquid-phase adsorption of dyes and phenols using pinewood-based activated carbons. Carbon. 2003:41(3):487-95.

50. Gonzo EE, Gonzo LF. Kinetics of phenol removal from aqueous solution by adsorption onto peanut shell acid-activated carbon. Adsorpt Sci Technol. 2005;23(4):289-302.

51. Cheung CW, Porter JF, Mckay G. Sorption kinetic analysis for the removal of cadmium ions from effluents using bone char. Water Res. 2001;35(3):605-12.

52. Villegas M, Castro Vidaurre EF, Habert AC, Gottifredi JC. Sorption and pervaporation with poly(3-hydroxybutyrate) membranes: methanol/methyl tert-butyl ether mixtures. J Memb Sci. 2011;367(1-2):103-9.

53. Carelli V, Di Colo G, Nannipieri E, Serafini MF. A study of controlled-release systems for progesterone based on crosslinked poly(ethylene oxides). Int J Pharm. 1993;94(1-3):103-13.

54. Cerchiara T, Luppi B, Bigucci F, Zecchi V. Effect of chitosan on progesterone release from hydroxypropyl- $\beta$-cyclodextrin complexes. Int J Pharm. 2003;258(1-2):209-15.

55. Liu Q, Wang X-Y, Zhang H. Solvent effects on infrared spectra of progesterone in $\mathrm{CHCl} /$ cyclo-C6H12 binary solvent systems. Spectrochim Acta, Part A. 2007;66(1):202-7.

56. Fini A, Ospitali F, Zoppetti G, Puppini N. ATR/Raman and fractal characterization of HPBCD/progesterone complex solid particles. Pharm Res. 2008;25(9):2030-40.

57. $\mathrm{Hu}$ Y, Zhang J, Sato H, Noda I, Ozaki Y. Multiple melting behavior of poly(3-hydroxybutyrate-co-3-hydroxyhexanoate) investigated by differential scanning calorimetry and infrared spectroscopy. Polymer. 2007;48(16):4777-85.

58. Nakanishi K, Solomon PH. Infrared absorption spectroscopy. 2nd ed. San Francisco, USA: Holden-Day Inc; 1977.

59. Benita S, Barkai A, Pathak YV. Effect of drug loading extent on the in vitro release kinetic behaviour of nifedipine from polyacrylate microspheres. J Control Release. 1990;12(3):213-22.

60. Grant DJW, Higuchi T. Chapter XI-Solubility behavior of organic compounds. Volume 21 of Techniques of chemistry. New York: John Wiley \& Sons; 1990 